\title{
COMPARISON OF ENCODING PROPERTIES OF CAMPANIFORM SENSILLA ON THE FLY WING
}

\author{
By MICHAEL H. DICKINSON* \\ Department of Zoology NJ-15, University of Washington, \\ Seattle, WA 98195, USA
}

Accepted 16 February 1990

\begin{abstract}
Summary
The wing blade of the blowfly Calliphora vomitoria (L.) carries an array of campaniform sensilla which have previously been divided into slowly and rapidly adapting classes based on their responses to step indentations. In the present study, the physiological characteristics of six sensilla of these two classes are examined within a $20-400 \mathrm{~Hz}$ frequency range, using a noise analysis that quantifies linear and nonlinear encoding properties. Both classes exhibit a broad response maximum near $150 \mathrm{~Hz}$, corresponding to the typical wingbeat frequency of the blowfly, and display rectification, limiting the spike response to a narrow portion of a stimulus cycle. The similarity in the encoding properties between the two groups is largely a consequence of the high wingbeat frequency of flies, which precludes any individual neurone from acting as a magnitude detector. Instead, during flight the campaniform neurones might act as 'one-shot' detectors, firing a single action potential at a precise phase of each wing stroke cycle. An array of such detectors would be capable of monitoring the passage of a deformational wave as it travels along the wing during each wingbeat.
\end{abstract}

\section{Introduction}

The organization of neurones within a sensory system into submodalities is a common feature of nervous systems (Young, 1989). Behaviourally relevant stimuli are often too complex to be adequately encoded by a single neurone type, and typically the individual cells within a sensory field are specialized to encode only a portion of the information contained in an external stimulus. One relatively simple example of physiological subclasses within a single sensory modality is the distal campaniform sensilla (strain detectors) on the wings of flies. In Drosophila, the projections of these mechanosensory neurones are segregated into two discrete pathways within the central nervous system (CNS) (medial tract and lateral tract) according to their adaptation rate and time of birth (Palka et al. 1986; Dickinson

* Present address: Department of Neuroscience, Roche Institute of Molecular Biology, 340 Kingsland Street, Nutley, NJ 07110, USA.

Key words: campaniform sensilla, fly wing, encoding properties. 
and Palka, 1987). The function of this central segregation of slowly and rapidly adapting campaniforms is unknown, but presumably reflects their behavioural roles. This paper examines the physiological characteristics of six of the homologous receptors on the wing of the blowfly Calliphora vomitoria, whose much larger size makes it possible to gain more insight into the possible roles of these neurones during flight. Mechanical noise stimulation techniques described in the first paper of this series (Dickinson, 1990) are used to quantify the responses of the campaniform cells within the frequency range that is likely to be relevant during flight. This analysis should be useful in unravelling the function of the two campaniform classes, as well as in determining those properties of wing deformation that may be important in flight.

\section{Materials and methods \\ Mechanical noise stimulation}

These experiments were conducted on 1- to 3-week-old blowflies, Calliphora vomitoria, from a laboratory population maintained on water, sucrose and powdered milk. The stimulus, recording and analysis methods have previously been described in detail (Dickinson, 1990) and are summarized briefly here. The basic approach was to stimulate identified campaniform sensilla with mechanical noise in order to calculate the gain and phase characteristics of the sensory neurones in the range of frequencies surrounding $150 \mathrm{~Hz}$, the typical wingbeat frequency of Calliphora. The domes of identified campaniform sensilla were mechanically stimulated with band-limited noise, eliciting spike trains from individual sensory neurones which were recorded with extracellular electrodes in saline pools contacting distal and proximal ends of an excised wing. The power level of the stimulus was determined prior to each experiment by varying the magnitude of a noise stimulus and choosing a stimulus level that resulted in a near maximal response. The spike rate, stimulus power and the pre-spike signal average of the stimulus were measured and used to calculate a function characterizing the linear behaviour of the neurone, $g(t)$. Another function, $m(\cdot)$, which accounts for the rectification of the sensory neurone, was computed by comparing actual responses elicited by noise stimuli with predictions generated by the linear function $g(t)$. Together, $g(t)$ and $m(\cdot)$ constitute a Wiener cascade model of the encoding properties of each campaniform neurone. The gain and phase response of a campaniform neurone may be computed by Fourier transformation of the $g(t)$ function.

The methods outlined above made possible the measurement or construction of the following: (1) campaniform dome compliance, which was subsequently used to compute indentation in each experiment on the six identified sensilla; (2) the relationship between stimulus power and spike rate, which was used to to estimate the functional stimulus range for a medial and a lateral tract sensillum (ACV and d-HCV); (3) functions representing linear and nonlinear encoding properties of 
the campaniform neurones. These functions were then used to reconstruct the frequency responses of specific sensilla and facilitate comparisons among them.

\section{Whole-wing deformations}

Stimulation of campaniforms by direct indentation of the sensilla domes is a convenient method for characterizing encoding properties, but in vivo dome indentation is caused by chordwise or torsional deformations of the wing. In flight, during the downstroke to upstroke transition when the leading edge is rapidly rotated upwards (supination), a distal to proximal torsional wave passes along the wing (Nachtigall, 1979; Wooton, 1981; Ellington, 1984; Ennos, 1988b, 1989). In order to examine the response of the wing sensilla to a more natural stimulus, the experimental -apparatus (Dickinson, 1990) was modified to allow torsional deformation of the wing while recording the responses of the campaniform neurones. In this configuration, only the base of the wing was fixed with cyanoacrylate, and the distal portion of the wing extended freely off the edge of the acrylic chamber. One electrode was placed in a saline pool that contacted the cut proximal portion of the wing. A fine steel pin was placed with a micromanipulator in the radial vein to provide the second electrode. The distal tip of the wing was clamped between two closely aligned insect pins, mounted on a torsion generator that provided twisting motion about the third wing vein, which contains most of the campaniform neurones. The torsion generator transformed the feedback-controlled vertical motion of a vibration generator into torsional movement. This stimulus arrangement mimicked in a simplified manner the torsional deformations that the campaniform sensilla might encounter during flight.

\section{Results}

\section{Compliance}

To compute the magnitude of campaniform dome indentation from the stimulus force record, it was necessary to measure the compliance of the dome at the end of each noise experiment. This measurement also provided one basis of comparison among the six closely analyzed sensilla (see Table 1). The compliances of all the sensilla were independent of frequency above $20 \mathrm{~Hz}$, and the value at $150 \mathrm{~Hz}\left(C_{150}\right)$ was used as a standard for comparison as it corresponds to the typical wingbeat frequency of Calliphora. The mean $C_{150}$ values for all six identified campaniforms fell between 0.7 and $1.0 \mathrm{~nm} \mu \mathrm{N}^{-1}$ (Table 1). These values are comparable to the compliance of the d-HCV sensillum on the fly wing (Dickinson, 1990) and the femoral campaniform sensilla of the cockroach (Chapman and Duckrow, 1975). There were no significant differences among the compliance values for any of the six sensilla, or between the medial and lateral tract classes (Student's $t$-test, $P>0.2$ in all cases). 


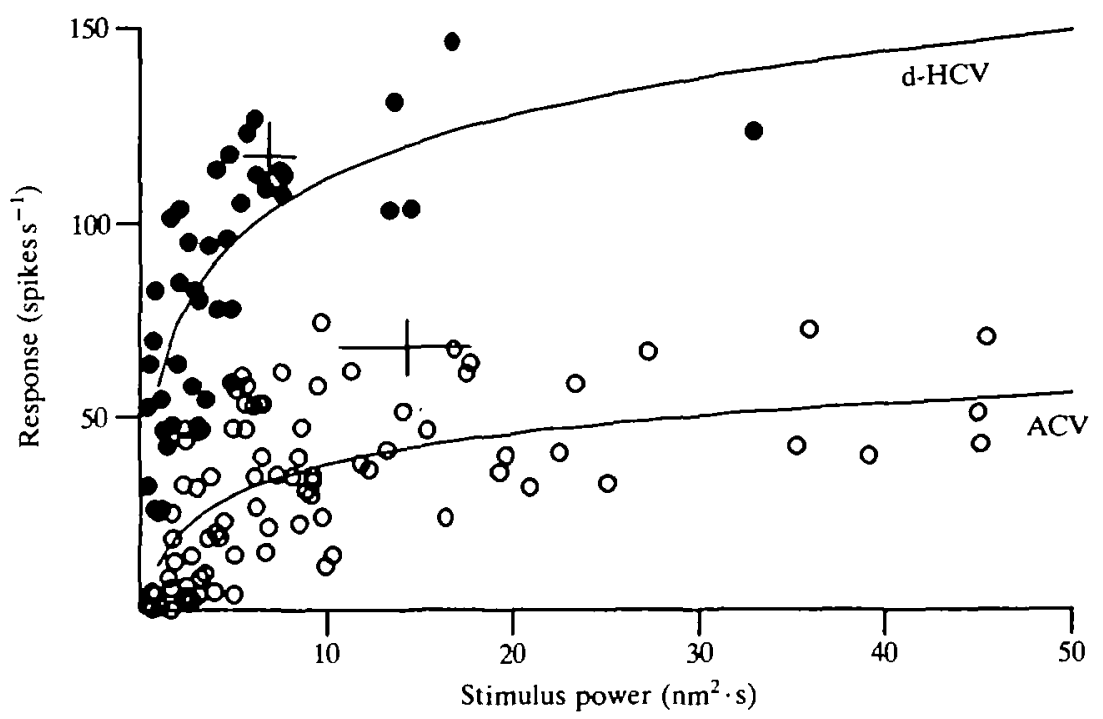

Fig. 1. Responses of d-HCV and ACV sensilla to increasing stimulus level. The mean spike rate during a $5 \mathrm{~s}$ mechanical noise stimulus is plotted as a function of power level. The data for the d-HCV (closed circles) have been replotted from Dickinson (1990, Fig. 5). Unlike the tonically responding d-HCV cell, the ACV is quiescent at low stimulus levels. In addition, the response of the d-HCV neurone reaches a higher level than that of the ACV. The two crosses show the mean stimulus levels and mean spike rates ( \pm s.D.) summed from all the separate noise stimulus experiments on the two neurones (see Table 1 of this paper, and Table 1, Dickinson, 1990). The solid lines are drawn through the equations $53.6+57.6 \log x(\mathrm{~d}-\mathrm{HCV})$ and $y=11.3+25.7 \log x(\mathrm{ACV})$, found by least-squares regression through the pooled data sets.

\section{Stimulus power and spike frequency}

Fig. 1 shows the effect of power level on spike rate over a wide stimulus range for two sensilla, the rapidly adapting $\mathrm{ACV}$ and the slowly adapting d-HCV (replotted from Dickinson, 1990). The data for the ACV have been pooled from eight different preparations. The stimulus-response relationships are approximated by the equations $y=53.6+57.6 \log x(\mathrm{~d}-\mathrm{HCV})$ and $y=11.3+25.7 \log x(\mathrm{ACV})$, fitted by least-squares regression. A tonic response resulting from the offset of the indentation stimulus is absent in the ACV neurone, which is consistent with its rapid adaptation rate, but is clearly seen in the $\mathrm{d}-\mathrm{HCV}$ cell as a residual spike rate at the lowest stimulus levels. Although the maximum spike rate is higher in the $\mathrm{d}-\mathrm{HCV}$ neurone, the absolute change in spike frequency above the tonic baseline, due to increasing levels of the noise stimulus, is of a similar magnitude in both neurones. The spike rate in both cells saturates and then remains relatively constant for large increases in power level, but the $\mathrm{d}-\mathrm{HCV}$ reaches saturation at a lower stimulus level than does the ACV.

The crosses in Fig. 1 indicate the stimulus power levels and spike rates (mean \pm s.D.) from the separate sets of experiments on the ACV and d-HCV 
Table 1. Comparisons of compliance, mean spike rate, stimulus power and sensitivity ( $\xi$ ) among identified campaniform neurones

\begin{tabular}{lcrcc}
\hline Cell & $\begin{array}{c}\text { Compliance } \\
\left(\mathrm{nm} \mu \mathrm{N}^{-1}\right)\end{array}$ & $\begin{array}{c}\text { Spike rate } \\
\left(\text { spikes s }^{-1}\right)\end{array}$ & $\begin{array}{c}\text { Power } \\
\left(\mathrm{nm}^{2} \mathrm{~s}\right)\end{array}$ & $\begin{array}{c}\xi \\
\left(\text { spikes s}^{-1}\right)\left(\mathrm{nm}^{2} \mathrm{~s}\right)^{-1}\end{array}$ \\
\hline Lateral tract neurones & & & & \\
p-TSM (7) & $0.74 \pm 0.21$ & $101.2 \pm 7.6$ & $3.8 \pm 0.8$ & $38.4 \pm 9.5$ \\
L3-1 (9) & $0.79 \pm 0.18$ & $73.6 \pm 6.8$ & $8.0 \pm 4.0$ & $28.9 \pm 8.8$ \\
L3-3 (8) & $0.73 \pm 0.14$ & $64.7 \pm 8.6$ & $6.4 \pm 2.0$ & $19.8 \pm 5.0$ \\
Medial tract neurones & & & \\
d-TSM (10) & $0.91 \pm 0.18$ & $48.0 \pm 3.9$ & $14.6 \pm 4.3$ & $6.4 \pm 1.4$ \\
ACV (15) & $0.91 \pm 0.13$ & $67.5 \pm 7.4$ & $14.2 \pm 3.6$ & $9.9 \pm 2.3$ \\
L3-2 (10) & $0.73 \pm 0.11$ & $40.6 \pm 8.1$ & $9.5 \pm 3.1$ & $8.7 \pm 2.7$ \\
& & & \\
Values are means \pm s.E.M. & & & \\
Number following cell name represents sample size. \\
Compliance was measured at a stimulus frequency of $150 \mathrm{~Hz}$.
\end{tabular}

(Table 1, and Table 1 of Dickinson, 1990) that were used to calculate the $g(t)$ and $m(\cdot)$ functions. The stimulus level in each of these experiments was set to produce a near maximal response in the campaniform neurone. The mean values used for the d-HCV correspond well with the data points from the four detailed experiments pooled in Fig. 1. Both the stimulus level and the resulting spike rates in the ACV experiments were slightly greater than expected from the sample data, but still lie within the range of variability for this neurone. Taken together, the data of Fig. 1 support the interpretation that the stimulus level chosen in each individual noise experiment roughly corresponds to the response shoulder where the spike rate begins to saturate.

The mean stimulus power levels and resulting spike rates from all the dome indentation experiments in this study are presented in Table 1 . The power levels varied among the six neurones, and stronger stimuli were generally required for the lateral tract class. Because the stimulus levels were empirically set to produce a near maximal response, these values reflect differences in the relationship between stimulus level and spike rate among the neurones. The lateral tract cells required lower power levels than the medial tract cells, suggesting that, like the d-HCV neurone, they saturate at lower stimulus intensities. With the exception of the L3-3 cell, the lateral tract neurones also displayed the highest mean spike rates, due in part to the presence of a tonic response caused by the stimulus offset.

\section{Linear and nonlinear encoding functions}

Measurements of $g(t)$, the first term of the Wiener cascade model, are compared for the six campaniform sensilla in Fig. 2. The function $g(t)$, which quantifies the linear encoding properties of the campaniform sensilla, is calculated by multiplying the stimulus pre-spike signal average by the ratio of mean spike rate to stimulus power. Each superimposed trace represents a measurement from a single 

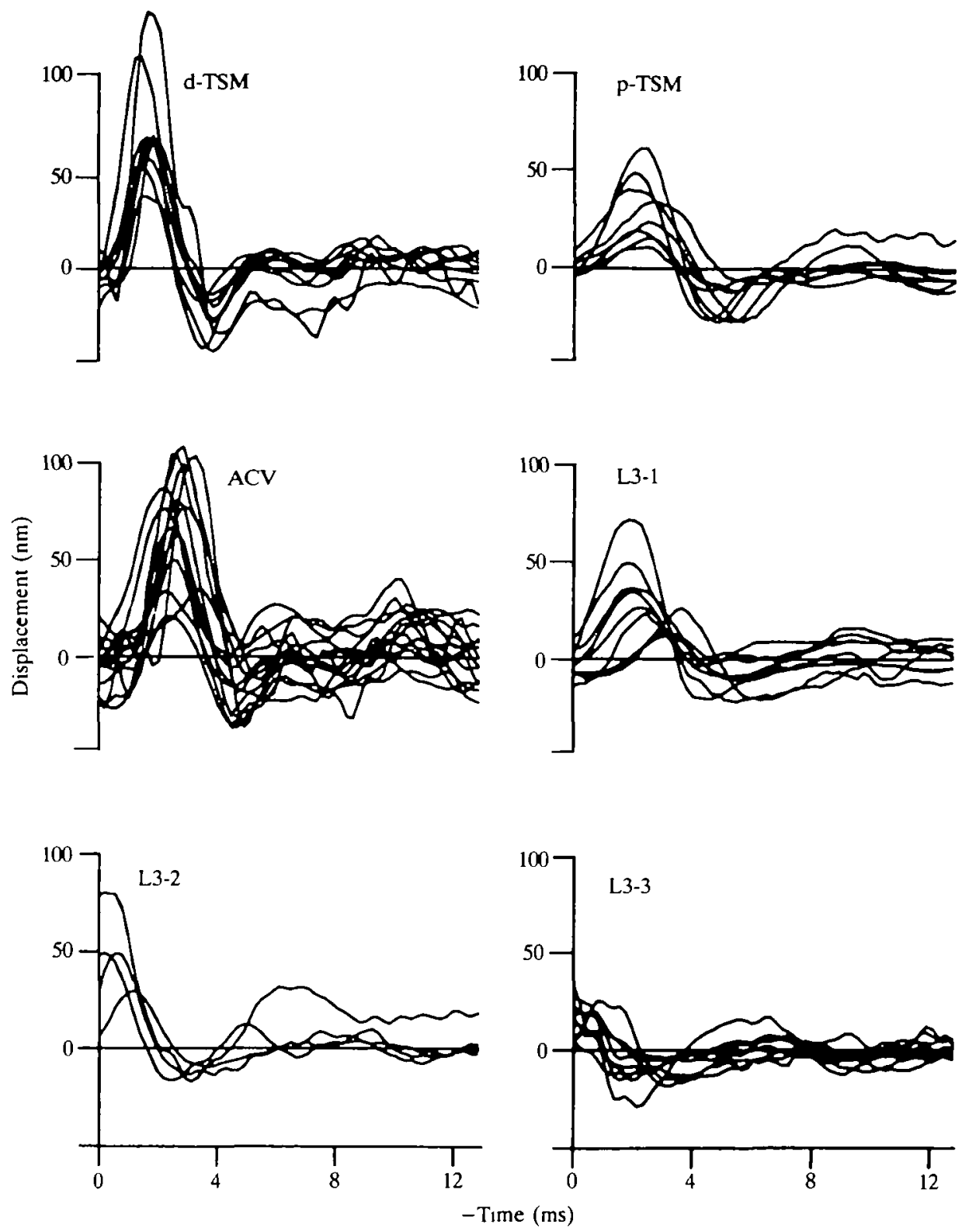

Fig. 2. Comparison of first Wiener cascade functions for six identified campaniform sensilla. Each panel contains superimposed traces of the averaged input function $g(t)$ calculated from corresponding sensilla from different preparations. There is variation in the replicate experiments, but the functions are quite different among the six cells.

preparation. Characteristic differences are apparent among the neurones which represent variations in the linear encoding properties. For all the neurones, the $g(t)$ functions approach 0 for values of $t$ greater than $6 \mathrm{~ms}$ before spike occurrence. 
This corresponds to a system memory of approximately one wing stroke period at a wingbeat frequency of $150 \mathrm{~Hz}$.

Because the sensilla are distributed widely along the third vein, it is crucial to determine if the recording geometry introduces conduction delays that might be responsible for the differences in the shapes of $g(t)$ among the campaniform sensilla. As argued in the first paper of this series (Dickinson, 1990), the conduction of the impulse down the axon does not produce any delay prior to the initial rise of the extracellular signal. However, in some preparations the trailing portion of the extracellular waveform of the most distal sensilla, L3-2 and L3-3, was contaminated by the propagated spike. Measurements of $g(t)$ and $m(\cdot)$ for these two sensilla are included only for those preparations in which the window discriminator circuitry could accurately detect the initial rise and ignore the subsequent components caused by the propagated spike.

Measurements of the static nonlinear function $m(\cdot)$ are shown in Fig. 3. These curves are derived from sixth-order polynomial fits of measured average responses to noise stimuli plotted against linear predictions calculated with $g(t)$ functions (see Dickinson, 1990). If the response of a campaniform neurone were perfectly predicted by the linear function $g(t)$, the function $m(\cdot)$ would have a slope of 1 . Thus, this function displays graphically any deviation from perfect linear encoding. The slope of the $m(\cdot)$ function in the right quadrant represents the degree of amplification. Rectification, which varies from cell to cell, is indicated by the decreased slope in the left quadrant, where the linear model predicts negative values of spike frequency. Because neurones cannot produce negative spike rates, linearity can only be achieved with a spontaneous discharge and low levels of amplification. The two most linear neurones, p-TSM and L3-1, display both these characteristics. The $m(\cdot)$ functions of the medial tract neurones, d-TSM, ACV and L3-2 all display sharper rectification and greater amplification than do the p-TSM and L3-1 cells. The L3-3 sensillum is the most variable, and displays a nonlinearity that is intermediate between the two classes.

\section{Frequency response}

The frequency responses of the neurones, derived by Fourier transformation of the linear functions $g(t)$ (Marmarelis, 1988), are shown in Fig. 4. The lateral tract neurones (open symbols) have higher gains than the medial tract cells (closed symbols) throughout most of the frequency range. The gains at $150 \mathrm{~Hz}$ (shown at the upper right of Fig. 4B) ranged between 2.1 (L3-2) and 5.1 (p-TSM) spikes $\mathrm{s}^{-1} \mathrm{~nm}^{-1}$. Thus, the least sensitive and the most sensitive campaniform neurones are separated by a mere 2.5 -fold difference in gain. With the exception of the d-TSM, which displays the strongest high-frequency behaviour, the peak responses of the campaniform neurones occur between 110 and $150 \mathrm{~Hz}$, suggesting that these cells are broadly tuned to the wingbeat frequency of Calliphora.

Fig. $4 \mathrm{C}$ plots the absolute time interval between peak indentation and peak 'response, calculated from Fourier transformations of the $g(t)$ functions. The intervals were calculated relative to the nearest peak stimulus, so that positive 

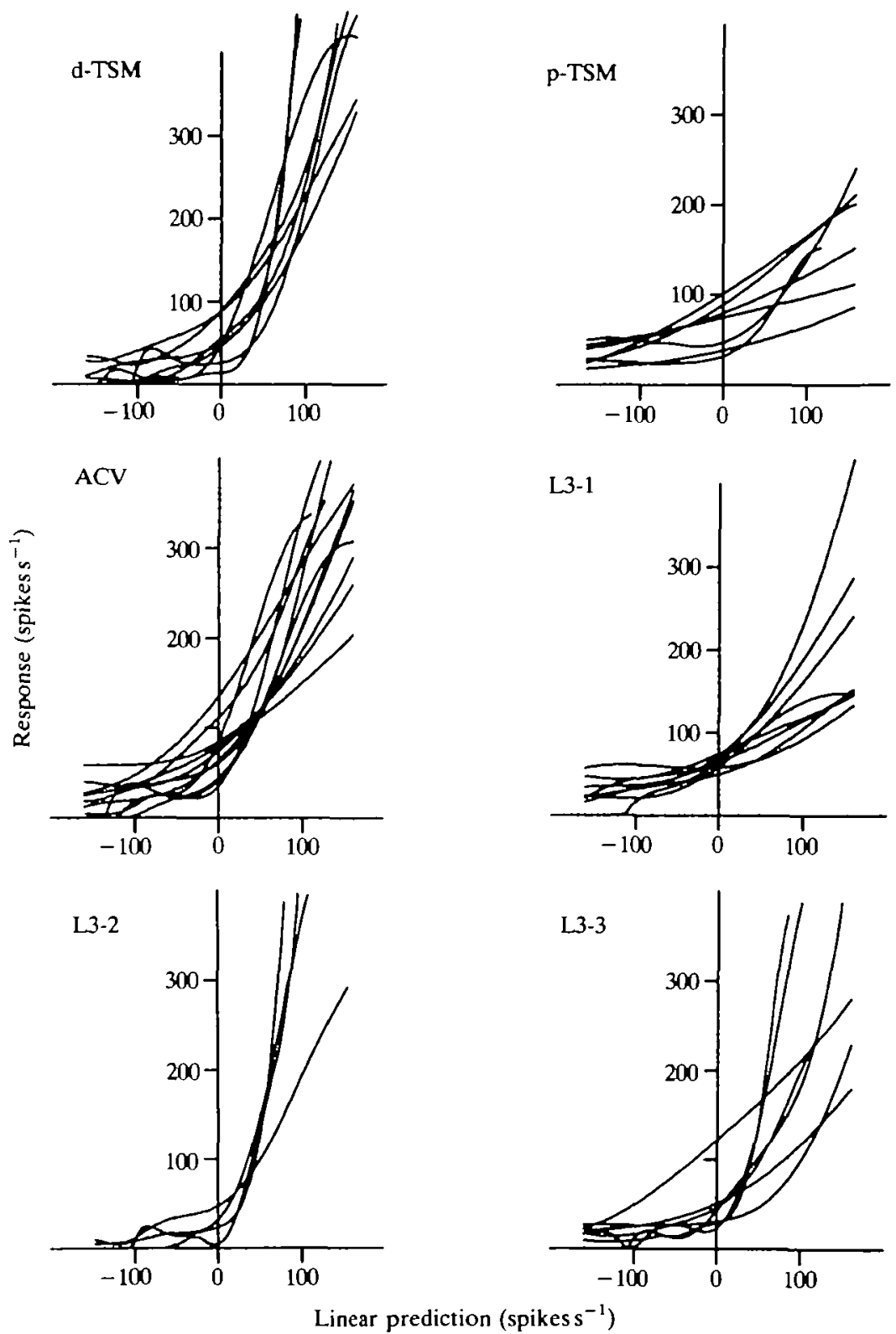

Fig. 3. Comparisons of the static nonlinear function $m(\cdot)$ for identified campaniform sensilla. The functions were constructed from a sixth-order polynomial fitted through a plot of the linear prediction, using only the $g(t)$ function, against the measured neurone response (see Dickinson, 1990). As in Fig. 2, each panel contains superimposed functions calculated from noise experiments on homologous neurones in different preparations. 
A
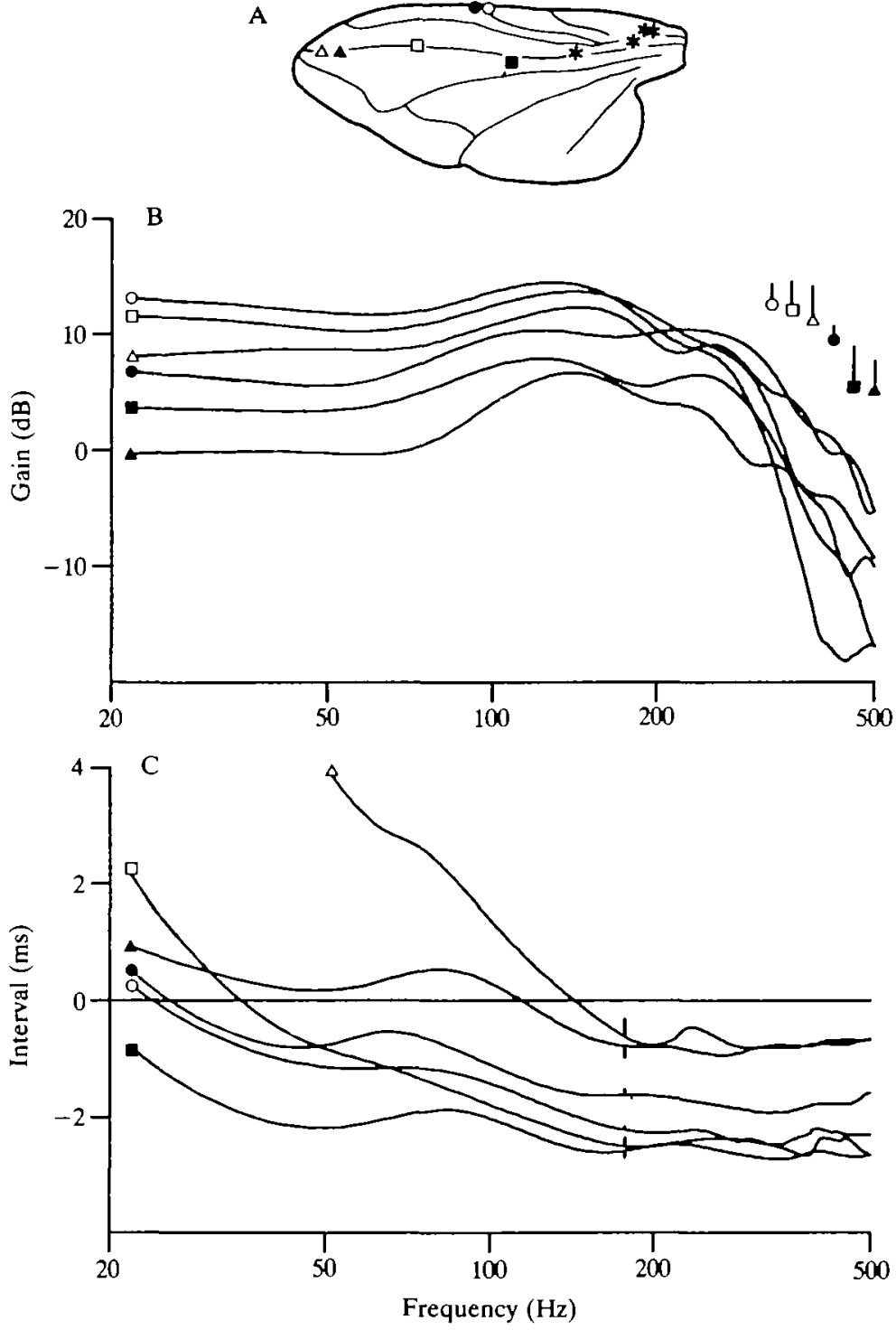

Fig. 4. Frequency response of campaniform sensilla. The frequency response of the campaniform neurones was calculated by transforming the Wiener cascade functions of Fig. 2 into the frequency domain. The campaniform neurones are identified by the symbols at the left of each trace (see wing map in A). (B) Gain calculated for campaniform neurones, plotted on a decibel scale. Each trace represents the means computed from each separate $g(t)$ function in Fig. 2. The symbols on the right of the figure display the mean value and S.E.M. of the gain calculated at $150 \mathrm{~Hz}$. (C) Phase relationships plotted in terms of time interval between peak response and nearest peak indentation. Positive values indicate leads, negative values indicate lags. Open circle, L3-1; open square, p-TSM; open triangle, L3-3 (medial tract cells); closed circle, d-TSM; closed square, ACV; closed triangle, L3-2 (lateral tract cells). 
values indicate response lead and negative values signify response lag. At frequencies above $50 \mathrm{~Hz}$, when all the neurones typically fire only a single spike for each stimulus cycle, this interval corresponds to the delay (or advance) between the peak dome indentation and the occurrence of a single action potential. At $150 \mathrm{~Hz}$, this delay varies between cells from roughly $-0.5 \mathrm{~ms}(\mathrm{~L} 3-3)$ to $-2.5 \mathrm{~ms}$ (ACV), or $8-38 \%$ of the wingbeat cycle.

The important results of Fig. 4 may be summarized by considering the expected responses of the campaniform neurones to sinusoidal wing oscillations. At frequencies over $50 \mathrm{~Hz}$, each neurone would fire a single action potential, with a stimulus amplitude threshold reflecting the differences in the sensitivity indicated in Fig. 4B. The interval between peak dome indentation and the single action potential would vary for each identified campaniform according to Fig. $4 \mathrm{C}$.

These predictions, based on the responses to punctate stimuli, can be verified by stimulating the campaniform neurones with whole-wing stimuli that more accurately mimic the deformations that are likely during flight. Fig. 5 shows the responses of campaniform sensilla to a $100 \mathrm{~Hz}$ torsion of the wing about its long axis. As the stimulus intensity is increased, more cells are recruited, each firing at a distinct phase of the stimulus. Subsequent ablations, produced by crushing the campaniforms (starting with the most distal) between sharp forceps, revealed that the largest spike in the records belonged to the d-TSM and the second largest unit was the ACV; the small unit could not be unambiguously identified. The magnitude of the torsional stimulus may have varied spatially, and it is therefore difficult to reconcile this recruitment pattern with the sensitivity differences derived from punctate stimulation experiments. In addition, the phase relationships between wing torsion and dome indentation are unknown, so it is not possible to relate the interval values of Fig. 4B to the delays seen in response to whole-wing deformation. Despite such limitations, however, these whole-wing stimulus experiments clearly indicate that under more natural stimulus conditions the wing campaniforms fire single action potentials at defined phases of each cycle and suggest that stimulus strength might be encoded by a recruitment process.

\section{Discussion}

\section{Comparison of medial and lateral tract classes}

Cobalt backfills of Calliphora wing nerves reveal medial and lateral tract projections similar to those published for Drosophila (Dickinson and Palka, 1986). The anatomy of individual campaniform neurones has never been investigated in Calliphora, but their assignment into medial tract and lateral tract classes is justified on the basis of very strong positional and physiological homology with Drosophila (Dickinson and Palka, 1987).

Considering the distinct differences in the tonic response of the medial and lateral tract neurones, it is surprising that their encoding properties at high frequencies are quite similar. All the campaniform neurones possess a response peak near $150 \mathrm{~Hz}$ (Fig. 4) and display a rectification that limits the window for 

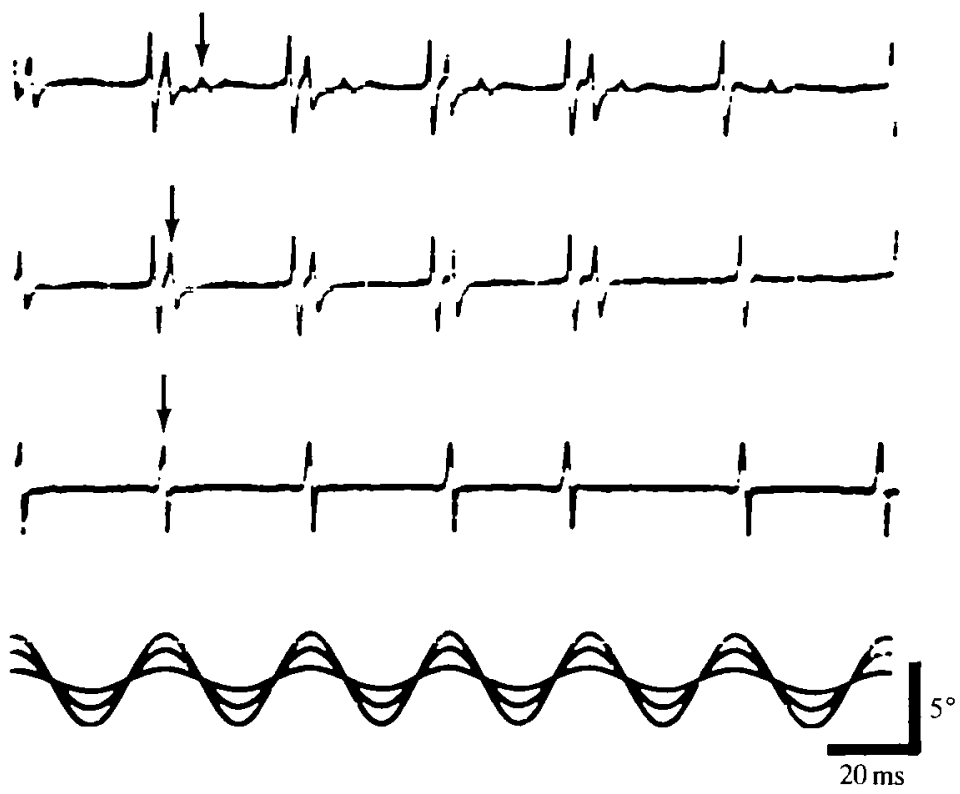

Fig. 5. Response of campaniform sensilla to torsional deformation of the wing. The base of the wing was fixed while the distal portion was oscillated at $100 \mathrm{~Hz}$. The wing was clamped just distal to the two campaniforms on the margin, p-TSM and d-TSM. Each of the top three traces shows a population recording of the wing campaniform sensilla, at different stimulus magnitudes. The smallest amplitude (bottom trace) elicits one large spike each cycle (arrow). Subsequent ablations identified this unit as the d-TSM. Increasing the stimulus amplitude recruits two more campaniform cells (arrows), each firing at a characteristic phase in the cycle. The second largest spike in the recordings belongs to the $\mathrm{ACV}$, but the small spike could not be unambiguously identified. Though the torsional stimulus presented is crude, this figure demonstrates how the campaniform sensilla might function during flight. The magnitudes of wing deformations are encoded by a recruitment process, and each cell fires at a characteristic time in the wingbeat cycle.

spike occurrence to a small interval (Figs 3,5). Any differences between the two classes are subtle: the medial tract neurones rectify more sharply than the lateral tract cells, and their responses extend to slightly higher frequencies. The lateral tract campaniforms are more sensitive throughout most of the frequency range. These differences in the high-frequency encoding properties are graded among all six neurones, however, and cannot be used to separate the two classes of cells, as can their tonic behaviour (Dickinson and Palka, 1987).

Despite these intrinsic similarities, there is likely to be a significant temporal spread in the arrival of action potentials in the central neuropile during each wingbeat cycle. The conduction time into the CNS must differ from cell to cell because the transit distance is much longer for the more distal cells and because the medial tract neurones possess larger axons (Dickinson and Palka, 1987). Wing deformations during flight have significant spatial and temporal components, and 
thus even neighbouring sensilla may not sense strains of identical magnitude or time course. In addition, the directional sensitivity of the cells will depend upon the eccentricity and orientation of the campaniform domes (Gnatzy et al. 1987), which has been shown in other preparations to bias strongly the responses of individual sensilla to cuticle deformations of a particular orientation (Zill et al. $1981 a, b)$.

The stimulus-response relationships of Fig. 1 suggest that neither medial nor lateral tract cell classes could function efficiently as magnitude detectors, contrary to what has been suggested for the wing campaniform sensilla of locusts (Elson, $1987 b$ ). The responses of both the d-HCV and the ACV saturate at a low power level and remain nearly constant with increasing stimulus strength. Although this relationship has to be interpreted cautiously in the absense of knowledge of the stimulus levels that occur during flight, these results are consistent with a 'oneshot' detector role for the campaniform neurones. Beyond a certain threshold, each campaniform neurone records one deformational event during each wingbeat, and further increases in stimulus strength do not change the pattern of firing. Such behaviour is seen in the whole-wing deformations of Fig. 5, where an increase in stimulus strength does not alter the firing pattern of the low-threshold cell, but instead recruits a greater number of campaniform neurones.

\section{Model for the function of the campaniform sensilla}

Fig. 6 shows diagrammatically a model for the function of the campaniform neurones that incorporates their intrinsic encoding characteristics together with the properties resulting from their spatial distribution on the wing. In the thoracic ganglia the campaniform neurones converge upon motor neurones of the direct flight muscles that control wing orientation. During a single wingbeat, the campaniform sensilla are stimulated by the distal-proximal deformational wave that is a characteristic feature of the wing kinematics of larger Diptera (Nachtigall, 1979; Wooton, 1981; Ellington, 1984; Ennos, 1988b, 1989). The strength of excitation produced centrally by the campaniform neurones depends upon the number of neurones responding and the time delays between arrivals of spikes at the synaptic sites. The recruitment and timing of spikes is in turn influenced by the magnitude and speed of the deformational wave on the wing. Based on Ennos' (1988b) ciné film tracings of free flight in a hoverfly Eristalis tenax, I estimate the speed of the torsional wave induced by supination to be approximately $10 \mathrm{~mm} \mathrm{~ms}^{-1}$, although the speed in Calliphora might be somewhat slower. Consider the L3-1 and L3-3 sensilla on the third vein, separated by about $3 \mathrm{~mm}$. The torsional wave passes these two campaniforms with roughly a $0.4 \mathrm{~ms}$ delay, or $6 \%$ of the wingstroke cycle at a wingbeat frequency of $150 \mathrm{~Hz}$. This delay would be greater for sensilla more widely spaced on the wing.

This model may explain why, in large calypterate flies, the L3-2 and L3-3 sensilla lie at the very distal end of the third vein near the wing tip (Dickinson and Palka, 1987). Based on the mechanical arguments of Ennos (1988 b), both bending and torsional torques decrease rapidly with distance from the wing base and thus the 


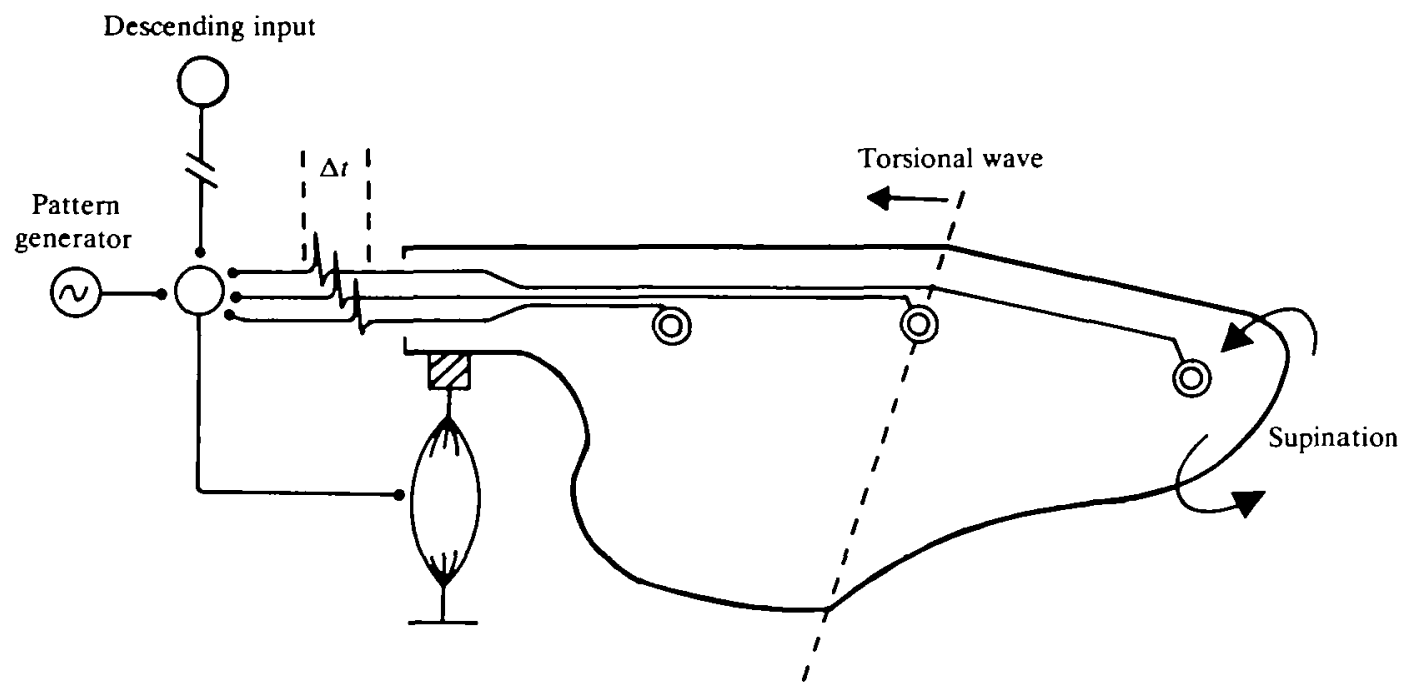

Fig. 6. Hypothesis for the function of large distal campaniform sensillum on the fly wing. Each sensilla is located at a stereotypic location on the wing surface. During a wingbeat, a torsional wave passes along the wing in a distal to proximal direction exciting the campaniform neurones. Each sensory neurone fires an action potential at a characteristic delay with respect to the stimulus. The input from the campaniform neurones converges upon central cells such as direct flight motor neurones. Temporal summation of campaniform input to the follower neurone will depend upon the spatial and temporal characteristics of the wing deformation during each wingbeat. The central targets of the campaniform cells also receive input from descending neurones and central and peripheral (sensory) elements of the flight pattern generator.

wing tip is not an efficient location for encoding the magnitude of these forces. However, if the campaniform sensilla function to encode the passage of torsional waves along the wing, this distal position will help to minimize the error in neural computations of transit speed by increasing the distance between proximal and distal sensilla.

Phase- and context-dependent gating of sensory information during flight is probably a general feature of insect flight systems, as has been demonstrated for locust steering (Reichert and Rowell, 1985) and fly head roll (Hengstenberg et al. 1986). The influence of the campaniform activity on follower neurones might also depend upon convergent elements of the pattern generator, including inputs from other phasic sensory modalities such as the halteres, as well as thoracic interneurones involved in the flight motor circuitry. In addition, the follower motor neurones might receive both phasic and tonic input descending from the head. Such convergence is found in locusts, where the central targets of the wing campaniform afferents receive convergent descending sensory input (Elson, 1987a).

Why might a fly be concerned with the subtleties of wing strain during flight? Wing deformations have been proposed to play important roles in the production 
of aerodynamic forces through changes in camber and angle of attack, the clapand-fling mechanism and vortex shedding (Vogel, 1967: Weis-Fogh, 1973; Wooton, 1981; Ellington, 1984; Ennos, 1988a). A recent theoretical study (Daniel, 1987) suggests that deformational waves of a particular magnitude and time course maximize the aerodynamic performance of a wing with a given shape. The system of campaniform neurones could provide an active feedback mechanism by which subtle characteristics of wing strain are encoded and are available to modulate the activity of the direct flight musculature.

\section{Could the activity in wing campaniform neurones be used to modify motor} activity on a wingbeat by wingbeat basis?

In the higher Diptera, which utilize an asynchronous flight motor (Pringle, 1957), phasic sensory modulation of the power musculature is probably not an important component of the flight pattern. In addition, the short wingbeat periods of most flies enforce a temporal limitation on sensory feedback circuits that could potentially operate within a single wing stroke. It is possible that the influence of the wing campaniforms is 'averaged' by the CNS over many wingbeat cycles, and influences only tonically active muscles. However, flies possess the necessary elements for a fast reflex, operating within a single wing stroke. Although most of the 17 pairs of direct flight muscles that have been investigated are active tonically during flight manoeuvres, at least one muscle, the first basalar (B1 in the nomenclature of Heide, 1971 $a$; Wisser and Nachtigall, 1984), is distinctive in that it fires a single phased-locked action potential for each wingbeat during flight (Heide, 1971b, 1983; Ewing, 1979) and during sine song (Ewing, 1979). Wingbeat synchronous afferents on the wings and halteres are responsible for this sharp phase tuning, since severing the wing and haltere nerves completely abolishes the phase-locked firing of the B1 during tethered flight (Heide, 1983). Of the many mechanosensory modalities that could be responsible for this influence, the large distal campaniform neurones on the wing are now the best characterized and appear well designed to entrain the B1 with high temporal precision. Cobalt backfills of the B1 motor neurone transynaptically fill both medial and lateral tract campaniform processes (M. H. Dickinson, unpublished observations). Cobalt coupling in Calliphora has indicated the presence of monosynaptic mixed electrotonic and chemical synapses between neurones (Bassemir and Strausfeld, 1983). Taken together this evidence suggests that certain aspects of the wing kinematics, influenced by the B1 muscle, could indeed be modulated by the phasic activity of the campaniform neurones on a cycle-by-cycle basis.

\section{Comparison of fly and locust wing campaniform sensilla and evolutionary implications}

The campaniform sensilla on the proximal portion of the locust wing share many similarities with fly wing sensilla. Although the physiological properties of single identified sensilla have not been determined, population recordings during wing torsion clearly show large and small units whose adaptation rates differ in a 
manner that is remarkably reminiscent of the rapidly and slowly adapting classes on the fly wing (Elson, 1987a). Elson (1987b) also demonstrated that, like the fly campaniforms, the locust sensilla must operate during flight at frequencies where constant delay dominates the phase behaviour. The sensilla provide afferent feedback to motor neurones and interneurones on a wing stroke by wing stroke basis, and are probably used in corrective behaviour (Horsmann and Wendler, 1985; Elson, 1987c). At frequencies typical of locust flight, many campaniform sensilla show little rate sensitivity and fire a series of action potentials during each cycle. Because of these properties, Elson (1987b) concluded that the locust wing campaniforms act primarily as magnitude detectors, firing a burst of action potentials during each cycle, whose strength depends on the magnitude of the wing torsion. Although the physiology of the locust and fly wing campaniforms is basically similar, the high wingbeat frequency of flies forces the sensilla into a different operating range. The fly neurones fire single spikes at each wingbeat, and therefore any individual campaniform sensillum does not make an efficient magnitude detector.

The wingbeat frequency in insects is inversely correlated with body size, and the wing campaniform neurones of large insects, like locusts, are more likely to operate in a frequency range where they can function as magnitude detectors. The campaniforms of smaller insects, with higher wingbeat frequencies, can only fire a single spike during each wing stroke, owing to constraints imposed ultimately by the kinetics of membrane properties. Primitively, wing deformations may have been encoded by both phasic and tonic receptors, the campaniform neurones of the medial and lateral tracts. In small insects with fast wingbeat frequencies, the distinction between the two neurone classes is diminished, and both act as 'oneshot' detectors, primarily concerned with the timing and not the magnitude of wing deformations in flight. Thus, the function of the sensilla has been qualitatively altered by an increase in wingbeat frequency, resulting ultimately from a size change. In this interpretation, evolutionary alterations in the function of campaniform sensilla may occur quite indirectly through selection on body size. This proposal is reminiscent of the size-dependent transition from a thermoregulative to an aerodynamic role for wings that has been proposed for the early stages of insect evolution (Kingsolver and Koehl, 1985).

Despite the convergence of high-frequency encoding properties between the two populations of campaniforms, it is likely that the tonic behaviour of the medial tract neurones still plays important behavioural roles. Besides flight, wings are used in other types of behaviour, such as courtship and grooming, in which the fly may need to monitor the magnitude of low-frequency deformations. The simple fact that the axons are segregated in the CNS presumably reflects some functional divergence between the two classes. Even during flight, tonically active neurones might be used to encode mean deformations of the wing, superimposed upon the fast torsional strains occurring during each wingbeat. Nevertheless, the convergence of high-frequency encoding behaviour between the campaniform neurones of two distinct physiological classes illustrates how functional changes can be 
imposed by alterations in size and wingbeat frequency, characteristics quite independent of the neurones themselves.

I wish to thank John Palka and Tom Daniel for their many helpful comments and suggestions with this manuscript. This work was supported by an NSF Graduate Fellowship, and NSF grant BNS-8507460 to John Palka.

\section{References}

Bassemir, U. K. and Strausfeld, N. J. (1983). Filled neurons in flies: cobalt deposits at presynaptic sites, mitochondria and the cytoskeleton. J. Neurocytol. 12, 949-970.

Chapman, K. M. and DuckCrow, R. B. (1975). Compliance and sensitivity of a mechanoreceptor of the insect integument. J. comp. Physiol. A 100, 251-268.

Daniel, T. L. (1987). Forward flapping flight from flexible fins. Can. J. Zool. 66, 630-638.

Dickinson, M. H. (1990). Linear and nonlinear encoding properties of an identified mechanoreceptor on the fly wing measured with mechanical noise stimuli. J. exp. Biol. 151, 219-244.

Dickinson, M. H. and PalKa, J. (1986). Homologous sensory neurons of the dipteran wing blade. Soc. Neurosci. Abstr. 12, 136.

Dickinson, M. H. And Palka, J. (1987). Physiological properties, time of development, and central projections are correlated in the wing mechanoreceptors of Drosophila. J. Neurosci. 7, $4201-4208$.

Ellington, C. P. (1984). The aerodynamics of hovering insect flight, III. Kinematics. Phil. Trans. R. Soc. Ser. B 305, 41-78.

ELSON, R. C. (1987a). Integration of wing proprioceptive and descending inputs by thoracic interneurones of the locust. J. exp. Biol. 128, 193-217.

Elson, R. C. (1987b). Flight motor neurone reflexes driven by strain-sensitive wing mechanoreceptors in the locust. J. comp. Physiol. A 161, 747-760.

ELSON, R. C. (1987c). Interneuronal processing of inputs from the campaniform sensilla of the locust hindwing. J. comp. Physiol. A 161, 761-776.

EnNos, A. R. (1988a). The importance of torsion in the design of insect wings. J. exp. Biol. 140, $137-160$.

EnNos, A. R. (1988b). The inertial cause of wing rotation in Diptera. J. exp. Biol. 140, 161-169.

Ennos, A. R. (1989). The kinematics and aerodynamics of the free flight of some Diptera. J. exp. Biol. 142, 49-85.

EwING, A. W. (1979). The role of feedback during singing and flight in Drosophila melanogaster. Physiol. Ent. 4, 329-337.

Gnatzy, W., Grúnert, U. AND Bender, M. (1987). Campaniform sensilla of Calliphora vicina (Insecta, Diptera). I. Topography. Zoomorphology 106, 312-319.

HeIDE, G. (1971a). Die Funktion der nicht-fibrillaren Flugmuskeln von Calliphora. Teil I. Lage, Insertionsstellen and Innervierungsmuster der Muskeln. Zool. Jb. Physiol. Bd. 76, 87-98.

HeIDE, G. (1971b). Die Funktion der nicht-fibrillären Flugmuskeln von Calliphora. Teil II. Muskulăre Mechanismen der Flugsteuerung und ihre nervöse Kontrolle. Zool. Jb. Physiol. Bd. 76, 99-137.

Heide, G. (1983). Neural mechanisms of flight control in Diptera. In Biona Report 2: Insect Flight (ed. W. Nachtigall), pp. 35-52. Stuttgart: Fischer.

Hengstenderg, R., Sandeman, D. C. and Hengstenberg, B. (1986). Compensatory head roll in the blowfly Calliphora during flight. Proc. R. Soc. B 227, 455-482.

Horsmann, U. and WendLer, G. (1985). The role of a fast wing reflex in locust flight. In Insect Locomotion (ed. M. Gewecke and G. Wendler), pp. 157-165. Berlin: Paul Parey.

Kingsolver, J. G. AND Koehl, M. A. R. (1985). Aerodynamics, thermoregulation, and the evolution of insect wings: differential scaling and evolutionary change. Evol. 39, 488-504.

Marmarelis, P. Z. (1988). Coherence and apparent transfer function measurements for nonlinear physiological systems. Ann. biomed. Eng. 16, 1-15. 
Marmarelis, P. Z. and Marmarelis, V. Z. (1978). Analysis Of Physiological Systems, The White Noise Approach. New York: Plenum Press.

Milsum, J. H. (1966). Biological Control System Analysis. New York, London: McGraw Hill.

Nachtigall, W.(1979). Rasche Richtungsänderungen und Torsionen schwingender Fliegenflügel und Hypothesen über zugeordnete instationäre Strömungseffekte. J. comp. Physiol. A 133, 351-355.

Palka, J., Malone, M. A., Ellison, R. L. and Wigston, D. J. (1986). Central projections of identified Drosophila sensory neurons in relation to their time of development. J. Neurosci. 6, $1822-1830$.

Pringle, J. W. S. B. (1957). Insect Flight. London: Cambridge University Press.

ReICHERT, H. AND Rowell, C. H. F. (1985). Integration of non-phaselocked exteroceptive information in the control of rythmic flight in the locust. $J$. Neurophysiol. 53, 1201-1218.

Vogel, S. (1967). Flight in Drosophila. III. Aerodynamic characteristics of fly wings and wing models. J. exp. Biol. 46, 431-443.

WeIs-FoGH, T. (1973). Quick estimates of flight fitness in hovering animals, including novel mechanisms for lift production. J. exp. Biol. 59, 169-230.

Wisser, A. AND NaChTIGALl, W. (1984). Functional-morphological investigations on the flight muscles and their insertion points in the blowfly Calliphora erythrocephala (Insecta, Diptera). Zoomorphology 104, 188-195.

Wooton, R. J. (1981). Support and deformability of insect wings. J. Zool., Lond. 193, 447-468.

Young, D. (1989). Nerve Cells and Animal Behaviour. Cambridge: Cambridge University Press.

ZiLL, S. N., Moran, D. T. ANd VARELA, F. G. (1981a). The exoskeleton and insect proprioception. I. Responses of tibial campaniform sensilla to external and muscle-generated forces in the American cockroach, Periplaneta americana. J. exp. Biol. 91, 1-24.

ZILL, S. N., Moran, D. T. ANd Varela, F. G. (1981b). The exoskeleton and insect proprioception. III. Activity of tibial campaniform sensilla during walking in the American cockroach, Periplaneta americana. J. exp. Biol. 94, 57-75. 